\title{
Geometric Registration for Zoomable Camera Using Epipolar Constraint and Pre-calibrated Intrinsic Camera Parameter Change
}

\author{
Takafumi Taketomi* Kazuya Okada $^{\dagger} \quad$ Goshiro Yamamoto Jun Miyazaki $^{\S}$ Hirokazu Kato ${ }^{\mathbb{I I}}$ \\ Nara Institute of Science and Technology, Japan
}

\begin{abstract}
In general, video see-through based augmented reality (AR) cannot change the magnification of camera zooming parameter due to the difficulty of dealing with changes in intrinsic camera parameters. To realize the usage of camera zooming in AR, we propose a novel simultaneous intrinsic and extrinsic camera parameter estimation method based on an energy minimization framework. Our method is composed of the online and offline stages. An intrinsic camera parameter change depending on the zoom values is calibrated in the offline stage. Intrinsic and extrinsic camera parameters are then estimated based on the energy minimization framework in the online stage. In our method, two energy terms are added to the conventional marker-based camera parameter estimation method. One is reprojection errors based on the epipolar constraint. The other is the constraint of continuity of zoom values. By using a novel energy function, our method can estimate accurate intrinsic and extrinsic camera parameters. In an experiment, we confirmed that the proposed method can achieve accurate camera parameter estimation during camera zooming.
\end{abstract}

Index Terms: H.5.1 [Multimedia Information Systems]: Artificial, augmented, and virtual realities; I.4.8 [Image Processing and Computer Vision]: Scene Analysis-Tracking; I.5.4 [Pattern Recognition]: Applications-Computer Vision

\section{INTRODUCTION}

In AR technology, camera parameter estimation is the most important technique to achieve geometric registration between real and virtual worlds. Generally, a marker-based camera parameter estimation method [3] is widely used in many applications because this method enable the easy construction of a robust AR environment. Marker-based methods assume that intrinsic camera parameters are fixed. However, cameras with zooming function have been used for AR applications. Zooming changes the intrinsic camera parameters. Removing this limitation of fixed intrinsic camera parameters in marker-based camera parameter estimation opens possibilities in many applications.

To solve this problem, Bujnak et al. propose the extrinsic camera parameter and focal length estimation method using Euclidean rigidity constraint in object space [1]. Furthermore, they improve the computational cost of the method [1] by joining planar and nonplanar solvers [2]. The method [2] can work in real-time in the desktop computer. However, the accuracy of estimated camera parameters still decreases in this method when the optical axis is perpendicular to the plane formed by the $3 \mathrm{D}$ points.

\footnotetext{
*e-mail: takafumi-t@is.naist.jp

†e-mail: kaduya-o@is.naist.jp

‡e-mail: goshiro@is.naist.jp

$\S$ e-mail: miyazaki@is.naist.jp

II e-mail: kato@is.naist.jp
}

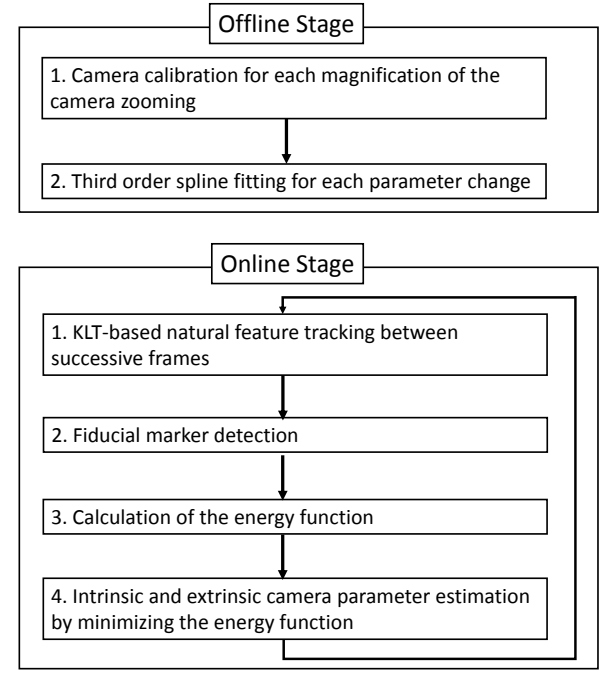

Figure 1: Flow diagram of the proposed method.

To realize simultaneous intrinsic and extrinsic camera parameter estimation while zooming, we propose a camera parameter estimation method using a pre-calibrated intrinsic camera parameter change and a novel energy function for the online camera parameter estimation. In our method, two energy terms are added to the conventional marker-based camera parameter estimation method: (1) Reprojection errors of tracked natural features and (2) Constraint of continuity of zoom values. By using the new energy function, our method can accurately and stably estimate intrinsic and extrinsic camera parameters in the online estimation process.

\section{Intrinsic and Extrinsic Camera Parameter Esti- MATION FOR ZOOMABLE CAMERA}

Our method is composed of the offline camera calibration and the online camera parameter estimation as shown in Fig. 1. Intrinsic camera parameters are modeled using a zoom variable in the calibration process, and then this model is used to estimate camera parameters in the online process. In the online process, several known 3-D points are used to estimate magnification of camera zooming and absolute extrinsic camera parameters.

\subsection{Parameterization of Intrinsic Camera Parameter with Zoom Value}

The relationship between magnification of camera zooming and intrinsic camera parameters is calibrated in the offline stage. In our method, for each magnification of the camera zooming, these four parameters are measured in an offline camera calibration process. Intrinsic camera parameters are then modeled by the magnification parameter of the camera zooming $m$. By using this parameterization, we can deal with the intrinsic camera parameter change using 
one parameter as shown in Equation (1).

$$
\boldsymbol{K}(m)=\left[\begin{array}{ccc}
f_{x}(m) & 0 & u(m) \\
0 & f_{y}(m) & v(m) \\
0 & 0 & 1
\end{array}\right]
$$

where $f_{x}$ and $f_{y}$ are focal lengths, and $u$ and $v$ are the center of projection.

In our method, third order spline fitting is used to obtain the model for each parameter change.

\subsection{Online Camera Parameter Estimation}

In the online stage, KLT-based natural feature tracking [4] and fiducial marker detection are executed. Camera parameters (translation, rotation, and magnification of camera zooming) are then estimated using these information. To estimate the intrinsic and extrinsic camera parameters in the online stage, we define the new energy function by adding the two energy term to the conventional markerbased camera parameter estimation. The energy function consists of three terms: (1) Reprojection errors of the fiducial marker $E_{m k}$, (2) Reprojection errors of tracked natural features based on epipolar constraint $E_{e p}$, and (3) Constraint of continuity of magnification of camera zooming $E_{z o o m}$. These three terms are automatically balanced using the weights $\omega_{m k}$ and $\omega_{z o o m}$ as follows.

$$
E^{2}=E_{e p}+\omega_{m k} E_{m k+}+\omega_{z o o m} E_{z o o m}
$$

$E_{m k}$ : Energy Term based on Fiducial Marker: This term is almost the same energy term of conventional camera parameter estimation methods. Reprojection errors are calculated from correspondences between fiducial marker corners in an input image and its reprojected points as follows.

$$
E_{m k}=\sum_{i=1}^{4}\left(\boldsymbol{K}\left(m_{j}\right) \boldsymbol{T}_{j} \boldsymbol{P}_{i}-\boldsymbol{p}_{i}^{\prime}\right)^{2}
$$

where $\boldsymbol{T}_{j}$ represents extrinsic camera parameter matrix composed of camera rotation and translation, and $\boldsymbol{P}_{i}$ and $\boldsymbol{p}_{i}{ }_{i}$ represent $3 \mathrm{D}$ position of fiducial marker corners and its detected position in the input image, respectively. Unlike the conventional methods, magnification parameter $m$ of the camera zooming exists in the intrinsic camera parameter matrix $\boldsymbol{K}$ in the $j$-th frame.

$E_{e p}$ : Energy Term based on Epipolar Constraint: $E_{e p}$ is calculated based on the epipolar constraint using tracked natural features between a key frame and a current frame.

Reprojection errors in term $E_{e p}$ is calculated using tracked natural features between a key frame and the input image. In the term $E_{e p}$, the reprojection error $d_{i}$ is defined as the distance between an epipolar line and a detected natural feature position in the input image as follows.

$$
E_{e p}=\frac{1}{\left|\boldsymbol{S}_{j}\right|} \sum_{i \in \boldsymbol{S}_{j}} d_{i}^{2}
$$

where $S$ represents a set of tracked natural feature points in the $j$-th frame. By using this notation, we can handle the estimation error for the two frames based on the epipolar constraint as the reprojection error.

$E_{z o o m}$ : Energy Term based on Continuity of Magnification of Camera Zooming: Our research is focused on the camera parameter estimation for augmented reality. Therefore, the online camera parameter estimation is executed sequentially. In this case, the magnification parameter of camera zooming within successive frames do not change drastically. To use this continuity constraint, we add the following energy term to $E_{\text {zoom }}$ :

$$
E_{z o o m}=\left(m_{j-1}-m_{j}\right)^{2}
$$

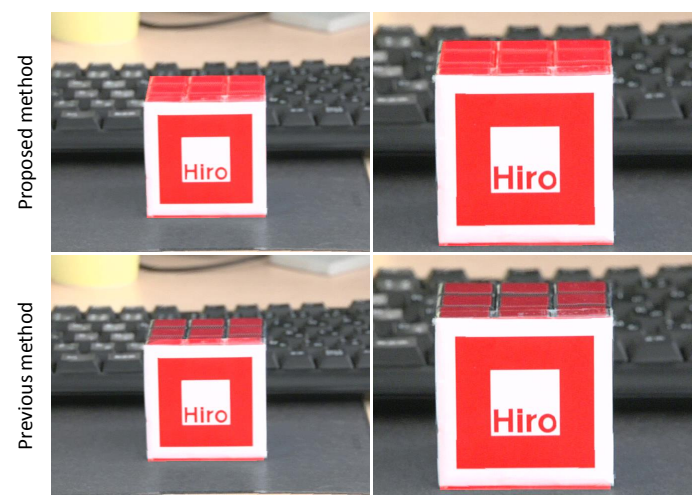

Figure 2: A virtual cube is overlaid to the Rubik's Cube in each frame.

With this constraint, a discontinuous change of the zoom value is suppressed.

Energy Minimization: To estimate the intrinsic and extrinsic camera parameters, the energy function $E$ is minimized by using the Levenberg-Marquardt algorithm. The M-estimator is employed in this optimization process to achieve the robust estimation. In this study, we employ the Geman-McClure function.

\section{EXPERIMENT}

To demonstrate the effectiveness of the proposed method, we compare the geometric registration result of our proposed method with that of the state-of-the-art method [2] which can handle the camera zooming.

Fig. 2 shows the result of the geometric registration. In this result, a virtual cube is overlaid to the Rubik's Cube. We can confirm that the virtual cube is accurately overlaid in the proposed method. In contrast, the results of the previous method involve geometric inconsistency. More specifically, there is large geometric inconsistency in the geometric registration result of the previous method for straight camera motion (Fig. 2). From these result our method can achieve accurate geometric registration using estimated camera parameters even in such a difficult condition.

\section{Conclusion}

In this paper, we proposed a intrinsic and extrinsic camera parameter estimation method for zoomable camera. To estimate intrinsic camera parameters while zooming, we have developed an energy function based on epipolar geometry. To achieve accurate camera parameter estimation, intrinsic camera parameters at each zoom value are calibrated in advance, and then the intrinsic camera parameter change depending on zooming is modeled. The effectiveness of the proposed method is demonstrated in the real environment. Currently, our method cannot deal with the lens distortion. In the future work, lens distortion estimation will be incorporated into the proposed method.

\section{REFERENCES}

[1] M. Bujnak, Z. Kukelova, and T. Pajdla. A general solution to the P4P problem for camera with unknown focal length. Proc. IEEE Conf. on Computer Vision and Pattern Recognition, pages 1-8, 2008.

[2] M. Bujnak, Z. Kukelova, and T. Pajdla. New efficient solution to the absolute pose problem for camera with unknown focal length and radial distortion. Proc. Asian Conf. on Computer Vision, pages 11-24, 2010.

[3] H. Kato and H. Billinghurst. Marker tracking and HMD calibration for a video-based augmented reality conferencing system. Proc. Int. Workshop on Augmented Reality, pages 85-94, 1999.

[4] J. Shi and C. Tomasi. Good features to track. Proc. IEEE Conf. on Computer Vision and Pattern Recognition, pages 593-600, 1994 